\title{
DIAGNOSIS AND TREATMENT IN GASTROESOPHAGEAL REFLUX DISEASE - POSSIBILITIES AND LIMITATIONS
}

\author{
Gabriela Ciubotariu', ${ }^{1}$ Smaranda Diaconescu, ${ }^{1,2}$, Angelica Cristina Marin', \\ Claudia Olaru ${ }^{1,2}$, Nicoleta Gimiga, ${ }^{1,2}$, Mihaela Moscalu ${ }^{2}$, Marin Burlea ${ }^{1,2}$ \\ I"Sf. Maria" Children's Hospital, Iasi \\ 2 "Gr. T. Popa” University of Medicine and Pharmacy, Iasi
}

\begin{abstract}
In children's first year of life it is mandatory to make a difference between physiological gastroesophageal reflux (GER) and gastroesophageal reflux disease (GERD), in order to avoid an aggressive pharmacologic therapy. All ages of childhood benefit from modern criteria of clinical evaluation, by corroborating the digestive or extradigestive symptoms with the results of modern investigations. The dietary measures (sufficient in GER but mandatory in all cases) together with proton pump inhibitors administration after one year of age induce remission in majority of GER cases. The chronic and recurrent evolution of the disease or failure of the common treatment require surgical intervention, recently enriched in children with peroral endoscopic myotomy (POEM).
\end{abstract}

Keywords: gastroesophageal reflux disease, proton pump inhibitors, Peroral Endoscopic Myotomy

\section{INTRODUCTION}

Gastroesophageal reflux (GERD) is physiological during the first 6-8 months of life and most of the times such episodes occur in the postprandial period, last up to 3 minutes and can be accompanied or not by symptoms. Over two thirds of the pediatric population is diagnosed with gastroesophageal reflux (1). The gastroesophageal reflux disease represents the totality of symptoms, accompanied or not by lesions of the esophageal mucosa caused by the contents of the stomach in the esophagus.

There is a low rate of GERD in breastfed children as opposed to those receiving milk formula. In this straightforward situation, the most important aspect refers to the manner in which the doctors and parents see the difference between physiological reflux and pathological reflux. The occurrence of GERD is influenced by both environmental factors and genetic factors, with the latter having an estimated $18-31 \%$ share in the onset of the disease.

The symptomatology associated with reflux has a high prevalence among the parents of the affected persons, and the correspondence is higher in the case of monozygotic twins as compared to heterozygotic twins (2).
Depending on the age, there are various typical clinical digestive signs (including regurgitation, vomiting, dysphagia, hematemesis and/or atypical extra-digestive signs. The atypical symptoms include laryngeal stridor, pharyngitis, coughing and/ or wheezing breath sounds, hoarseness, otitis media, non-cardiac chest pain, and dental erosions. Rumination is frequently noticed in premature infants (3).

Infants up to the age of one can present postprandial regurgitations, irritability, anorexia and/or refusal of food, growth failure, dysphagia or arching of the back during feeding. Regurgitation episodes can be postprandial or can occur within the 1-2 hours postprandial period $(4,5)$. The manifestations of GERD in children aged between 1 and 5 years include regurgitations, postprandial vomiting, abdominal pain, anorexia and refusal of food, while children over the age of 5 and teenagers describe the symptoms of gastroesophageal reflux disease just like adults do: pyrosis, epigastralgia, non-cardiac chest pain, especially during the night, and acid eructation (5).

There is a high risk of GERD onset in children with neurological disorders, obesity, histories of esophageal atresia (surgically treated), hiatal hernia, achalasia cardia, bronchopulmonary dysplasia,

Corresponding author:

Lecturer Smaranda Diaconescu MD, PhD, “Gr. T. Popa” University of Medicine and Pharmacy, 16 Universitatii Street, lasi

E-mail: turti23@yahoo.com 
cystic fibrosis, lung transplant, or premature infants (5).

GER is an aggravating factor for atopic or nonatopic bronchial asthma because microaspirations of gastric refluxate cause bronchoconstrion. The typical symptoms of GER are found in $45 \%$ of the chronic asthma patient lots as opposed to $10 \%$ in population-wide studies (5).

Other causes of vomiting include: congenital malformations of the esophagus, allergy to cow's milk protein and metabolic disorders.

In general, physiological gastroesophageal reflux can cause various symptoms without interfering with the child's growth and development. In GERD, the occurrence of reflux esophagitis manifests itself as aversion to food due to the pain caused by feeding, as well as the stopping or dropping of the weight curve.

The Chicago Classification (2008) provided further specifications regarding the diagnosis of the disease and the assessment of the response to treatment. During the initial assessment, the physician has to take an accurate and detailed history of the typical and atypical symptoms of GERD, their severity and previous courses of treatment.

The Eckardt score offers a quantitative assessment of symptoms severity, as well as the presence and frequency of dysphagia, regurgitations and weight loss (6).

Although the decrease of the LES pressure is the main cause of GERD, the single most important factor in the pathogenesis of reflux esophagitis is the recurring transient and inadequate relaxation of the LES. In addition to acid and non-acid reflux, the inflammation of the squamous esophageal epithelium can be associated with dysmotility, infections and food allergies.

First, there is basal cells hyperplasia and thickening of the papillae, followed by the inflammatory cells infiltration, ulcerations, and fibrosis with stenosis. Columnar metaplasia, also known as Barrett's esophagus, is a complication of GERD with a high risk of adenocarcinoma that rarely occurs in pediatric patients (5).

The neurobehavioral manifestations were attributed to the perception of pain and are characterized by: sleep disorders, agitation and crying fits, arching and rigidity, hyperextension of the neck, generalized irritability, sometimes with seizures or pseudo-psychotic behavior. The hypersensitivity of the components of the pain matrix associated with the functional disruption of cognitive and emotional centers is an important factor in the perpetuation of pain (weeks, years). There are associated modifica- tions in the autonomic nervous system and the esophageal motility, which determine the visceral hyperalgesia (7).

The Sandifer syndrome is a less frequent clinical entity, that is often mistaken for an epileptic seizure associating GER with head extension, torticollis, vicious position, opisthotonus, facial asymmetry and esophagitis related lesions.

The case history and clinical exam are essential in order to rule out other disease that can be associated with vomiting.

A history of regurgitations or vomiting episodes, ponderal stagnation and microcytic hypochromic anemia should alert both the parents and the treating physician for the purpose of developing a GER investigations protocol.

Depending on the seriousness, the international guidelines recommend the following GER exploration protocol: monitoring of esophageal $\mathrm{pH}$, esophageal manometry, upper gastrointestinal endoscopy, esogastric barium meal, and intraluminal esophageal impedance.

Monitoring of the esophageal $\mathrm{pH}$ means measuring the quantity of acid that the esophagus is exposed to over preset time intervals (quantitative testing). The severity of pathologic acid reflux is not correlated with that of the GER symptoms or complications. The clinical specificity, sensitivity and utility of esophageal $\mathrm{pH}$ monitoring for developing the diagnosis and management protocol for GERD complications has yet to be established $(8,9)$.

Esophageal manometry can be positive for GERD patients, but the results have no specificity or sensitivity for diagnosis confirmation purposes. This investigation allows to establish the diagnosis of a motility disorder or to confirm the achalasia cardia diagnosis $(8,9)$.

Upper gastrointestinal endoscopy (UGIE) is indicated for patients that are unresponsive to treatment. UGIE highlights ulcerations, erosions and esophageal strictures, and allows the taking of a biopsy for histopathological examination purposes, being an important factor in the identification of causes for esophagitis or the diagnosis of Helicobacter Pylori (H.P) infection. The relation between the H.P. infection and GERD remains uncertain, with some authors stating that the fact that $\mathrm{H}$. pylori infection favors inflammatory lesions paradoxically contributes to the good functioning of the anti-reflux barrier at the esogastric junction level $(10,11)$. Other authors suggest that the H. pylori infection determines the occurrence of inflammatory lesions, inclusively at the level of the cardia, thus favoring gastroesophageal reflux $(10,11)$. 
Esogastric barium meal provides information on the existence of hiatal hernias, secondary esophageal stenosis or dysmotilities of the esophagus, particularly after direct anastomosis for esophageal atresia. There are cases when in spite of the absence of symptoms this imaging exam reveals cases of GERD, and $60 \%$ of the patients with reflux symptoms and $\mathrm{pH}$-metric results conclusive for reflux showed no traces of reflux in the imaging examination $(2,3)$.

Intraluminal esophageal impedance detects both acid and no-acid reflux, being a modern investigative technique for the stomach and esophageal $\mathrm{pH}$. Depending on the type of attached electrode, the method can record the $\mathrm{pH}$ at one or several levels (proximal esophagus, distal esophagus, lower esophageal sphincter, stomach) or it can be used for simultaneous recording of pressures within the upper gastrointestinal tract, obtaining a quantitative assessment of how serious the gastric reflux is $(3,12)$.

Complete blood count and the value of serum iron are frequently modifies in case of GERD, but their decrease can be a result of other diseases, which requires differential diagnosis.

The exploration of patients with suspected GERD can be complex due to the evolution of particular comorbidities into predominantly extra-digestive manifestations (recurrent wheezing, serous otitis, recurrent laryngitis).

The treatment for gastroesophageal reflux disease entails a series of hygienic-dietary and pharmacotherapeutic measures depending on the child's age.

Upon demand, in the case of breastfed infants, care will be taken in order to avoid postprandial dorsal decubitus; in the case of formula-fed infants, care will be taken to observe a feeding schedule and avoid excessive feeding, gradually introducing the night-time feeding pause. The following measures shall be applied for children aged 1-5 years old (as necessary): avoiding dorsal decubitus for at least a 1-3 hours postprandial period, avoiding heavy meals, avoiding the proclive position (at a $45^{\circ}$ angle) both during the awake state and during sleep, considering the fact that after the age of 1 the risk of sudden death is greatly reduced (13). The recommendation in the case of older children and teenagers is to avoid triggering factors: caffeine, chocolate, spicy foods, fizzy soft drinks, mint and tobacco (4). Recent studies show that sugar-free chewing gum helps reduce reflux episodes (4).

The objectives of the pharmacologic treatment focus on symptoms' control, prevention and healing of esophagitis or other complications.
The persistence of the symptomatology in spite of an adequate hygienic-dietary treatment entails the initiation of medicine-based treatment, differentiated depending on the presence of complications.

Pharmacologic interventions seek to reduce the number and duration of reflux episodes, to restore the normal pressure of the LES, to improve esophageal clearance and to reduce the gastric emptying time. The accumulated expertise in treating GERD enforced a triple therapy: prokinetics, antisecretory drugs and antacids, administered in 8-weeks courses.

Recent studies have shown that this method helps reduce acidity, but not the total number of reflux episodes (14).

Prokinetics are administered in order to increase the pressure of the lower esophageal sphincter (LES), to stimulate esophageal peristalsis and to accelerate gastric emptying.

Recommended drugs include domperidone, benzimidazole derivative, dopamine antagonists, which act outside the central nervous system. The dose is $0.75-1 \mathrm{mg} / \mathrm{kg} / \mathrm{day}$, in 2 rounds, $30-45 \mathrm{~min}-$ utes before the meal.

Antisecretory medication is an important adjuvant for the prokinetic treatment, recommended particularly for patients with lesions caused by esophagitis.

Proton-pump inhibitors (PPIs) is the most effective treatment method, leading to the suppression of hydrochloric acid, the deactivation of proton pumps in parietal cells and the reduction of secretion; they eliminate the reflux symptomatology in over $90 \%$ of cases. The course of treatment is followed through for a period of 4-8 weeks. Current diagnosis and treatment guidelines recommend increasing the doses of PPIs up to $1.5-2 \mathrm{mg} / \mathrm{kg} / \mathrm{day}$ (1). PPIs shall not be administered to infants under the age of 1 .

Antacids can be used in mild cases, with rare reflux episodes and no esophagitis lesions.

Confirmation of the chronic recurrent GERD diagnosis and its resistance to treatment leads to surgical treatment.

The indications of surgical treatment include: persistent symptomatology, long term medicine dependence, noncompliance with the medicine-based therapy or aspiration pneumonia.

The parents' informed consent is compulsory due to possible postoperative complications, including recurrence of the symptomatology.

The standard surgical treatment is the Nissen fundoplication, performed under general anesthe- 
sia and using the classical surgical protocol; laparoscopic interventions have recently become more frequently used as they are less invasive and less aggressive. The doctor will have to take an accurate case history in terms of the number of previous abdominal and thoracic surgical interventions, because the laparoscopic intervention could not be indicated for this purpose. Immediate postoperative complications include dysphagia, precocious satiety or nausea. Delayed complications can include gastrointestinal stasis, herniation of the lining, intestinal adhesions or obstructions (15).

Endoluminal therapy seeks to inhibit the reflux at the level of the LES in order to lower the frequency of acid overflows into the esophagus. The Stretta procedure (radiofrequency energy) is recommended in GERD patients that are unresponsive to or intolerant of drug treatment. The delivery of radiofrequency energy at the level of the cardia is associated with an increased pressure of the LES and a lower number of transient relaxations of the sphincter, with an improvement in terms of quality of life over 6 months as of applying this procedure.

The creation of a thickening of the gastroesophageal junction by performing an endoscopic plication technique (Endocinch) or by resorting to the plicator for the entire thickness of the wall (NDO), obtaining a serous-to-serous tissue bond, is aimed at restoring and consolidating the valve mechanism of the esogastric junction (16).

Peroral Endoscopic Myotomy (POEM) is a minimally invasive technique, performed in patients with cardia achalasia. Achalasia is defined by the ineffectiveness of the LES resulting in differences in the pressures of the latter. (17) From a clinical point of view, it is accompanied by a polymorphous symptomatology: regurgitations, dysphagia, retro-sternal pain, and weight loss. POEM was initially described by Heller in 1913, consisting of 2 longitudinal incisions of approximately 8 $\mathrm{cm}$ on the anterior and posterior walls of the esophagus, for the purpose of releasing the pressure of the LES in order to completely improve the symptoms of achalasia. The limitation and failure of this technique were caused by gastroesophageal reflux, which was reported in $30 \%$ of the cases, thus re- quiring an additional anti-reflux procedure, namely the Nissen fundoplication.

There were three reported cases diagnosed with cardia achalasia that were subjected to this minimally invasive surgical method. The average age was 9.6 years. The average value of the LES basal pressure and Eckardt score were $34.6 \mathrm{mmHg}$ and 5.3 , respectively. The intervention was carried out over an average $10 \mathrm{~cm}$ length and had a duration of 60.6 minutes. No postoperative complications or GERD were reported. At the 1 year follow-up, the Eckardt score was 0, and the pressure of the LES showed a significant decrease (18).

Papasavas PK makes a retrospective analysis of the results of the POEM technique as applied in 15 children diagnosed with cardia achalasia upon performance of manometry, barium meal and UGIE:

- the average surgical time was 100 minutes (38-240'), postoperative reduction of the LES pressure from $36.64 \pm 11.08 \mathrm{mmHg}$ to $15.65 \pm 5.73 \mathrm{mmHg}$;

- clinical success was determined according to the value of the Eckardt score, below 3;

- ten of the fifteen children reported to the 1 year follow-up.

The average Eckardt scores preoperatively and postoperatively was $7.32 \pm 1.42$ and $1.74 \pm 0.67$, respectively, a result that was accompanied by an improvement in terms of symptomatology and a weight gain in the children by $0.46 \mathrm{~g} / \mathrm{year}(0.0-4.6$ interval) (19).

\section{CONCLUSIONS}

Gastroesophageal reflux disease has a complex clinical picture, with modern diagnosis methods revealing the actual incidence rate of the disease. Proton pump inhibitors become the first option in terms of drug therapy for children over the age of 1 .

Monitoring the esophageal $\mathrm{pH}$ remains a low cost investigation method with a broad spectrum of applicability, as well as entailing minimum complications. Cases that are unresponsive to drug treatments are often subjected to surgical interventions. The POEM technique proves to be innovative both in terms of its minimally invasive approach and its lack of complications.

\section{REFERENCES}

1. Vandenplas Y., Rudolph C.D., Di Lorenzo C., et al. North American Society for Pediatric Gastroenterology, Hepatology, and Nutrition; European Society for Pediatric Gastroenterology, Hepatology, and Nutrition. Pediatric gastroesophageal reflux clinical practice guidelines: joint recommendations of the North American Society for Pediatric Gastroenterology, Hepatology, and Nutrition (NASPGHAN) and the European Society for Pediatric Gastroenterology, Hepatology, and Nutrition (ESPGHAN). J Pediatr Gastroenterol Nutr. 2009; 49(4):498-547. 
2. Scurtu $A$. Rolul polimorfismului genetic în geneza bolii de reflux gastroesofagian. Arta Medica, 2/2015: CZU: 575:616.329-008.1, p 33-35.

3. Schwarz S., Hebra A. Pediatric gastroesophageal reflux. Medscape. http://emedicine.medscape.com/article/930029-overview. Accessed July 24, 2015.

4. Avidan B., Sonnenberg A., Schnell T.G., Sontag S.J. Walking and chewing reduce postprandial acid reflux. Aliment Pharmacol Ther. 2001; 15(2):151-155.

5. Lightdale J., Gremse D. Gastroesophageal reflux: management guidance for the pediatrician. Pediatrics. 2013; 131(5):e1684-e1695. http://pediatrics.aappublications.org/content/131/5/e1684.full. Accessed July 24, 2016.

6. Fisichella P.M., Jalilvand A., Leventhal A. Diagnostic Evaluation of Achalasia From the Whalebone to the Chicago Classification. World Journal of Surgery, January 2015, 39(7).

7. Ioniuc I., Alexoaie M., Russu G. Durerea abdominală nonorganică şi hiperalgezia viscerală. Revista Română de Pediatrie 2013,62,4: 350-410.

8. Loots C.M., Benninga M.A., Davidson G.P., et al. Addition of pHimpedance monitoring to standard $\mathrm{pH}$ monitoring increases the yield of symptom association analysis in infants and children with gastroesophageal reflux. J Pediatr 2009; 154:248-52

9. Shin M.S. Esophageal $\mathrm{pH}$ and Combined Impedance-pH Monitoring in Children. Pediatric Gastroenterology, Hepatology \& Nutrition. 2014; 17(1):13-22. doi:10.5223/pghn.2014.17.1.13.

10. Richter Joel E., Falk Gary W. et al. Helicobacter pylori and gastroesophageal reflux disease: The Berg May Not Be All Bad, Am.J. of Gastroenterol. 1998; vol.93, no. 10, 1800-1801.

11. Labenz J., Malfertheimer P. Helicobacter pylori in gastroesophageal reflux disease: causal agent, independent or protective factor? Gut 1997, 41, 277-280
12. Shay S., Tutuian R., Sifrim D., et al. Twenty-four hour ambulatory simultaneous impedance and $\mathrm{pH}$ monitoring: a multicenter report of normal values from 60 healthy volunteers. Am J Gastroenterol. 2004; 99(6):1037-1043.

13. Corvaglia L., Martini S., Aceti A., Arcuri S., Rossini R., Faldella G. Nonpharmacological Management of Gastroesophageal Reflux in Preterm Infants. Biomed Res. Int 2013: 141967.

14. Lupu V.V., Diaconescu S., Burlea M. Tratamentul bolii de reflux gastroesofagian la copil. Revista Română de Pediatrie. Nr 4/2010, p. 262-267.

15. Fonkalsurd E.W., Ashcraft K.W., Coran A.G. Surgical Treatment of gastroesophageal reflux in children; Pediatrics March 1998, 101 (3): 419-422.

16. Toquati A., Houston H.L., Kaiser J., Holzman M.D., Richard W.O. Long-term follow-up study of the Stretta procedure for the treatment of gastroesophageal reflux disease. Surg. Endosc. 2004 Oct; 18(10):1475-9.Epub2004 Joule 22.

17. Nabi Z., Ramchandani M., Reddy D.N., Darisetty S., Kotla R., Kalapala R., Chavan R. Per-oral Endoscopic Myotomy in Children with Achalasia Cardia. J Neurogastroenterol Motil. 2016 Apr 2.

18. Familiari P., Marchese M., Gigante G.I., Tringali A., Perri V., Costamagna G. Peroral Endoscopic Myotomy for the Treatment of Achalasia in Children, Journal of Pediatric Gastroenerology\&Nutrition: December 2013-Volume57-Issue 6-p794-797.

19. Papasavas P.K., Keenan R.J. et al. Effectiveness of laparoscopic fundoplication in relieving the symptoms of gastroesophageal reflux disease (GERD) and eliminating antireflux medical therapy. Surgical Endoscopy. 2003. 17. 1200-1205. 